PROCEEDINGS OF THE

AMERICAN MATHEMATICAL SOCIETY

Volume 126, Number 5, May 1998, Pages 1565-1571

S $0002-9939(98) 04181-1$

\title{
THE DETERMINATION OF THE PAIRS OF TWO-BRIDGE KNOTS OR LINKS WITH GORDIAN DISTANCE ONE
}

\author{
ICHIRO TORISU
}

(Communicated by Ronald A. Fintushel)

\begin{abstract}
We thoroughly determine the pairs of two-bridge knots or links with Gordian distance one. In addition, we examine the Gordian distance between a Montesinos knot (or link) and a two-bridge knot (or link).
\end{abstract}

\section{INTRODUCTION}

For any two knots or links $K, K^{\prime}$ in $S^{3}$, we can define the Gordian distance from $K$ to $K^{\prime}$, denoted by $d_{G}\left(K, K^{\prime}\right)$, to be the minimal number of crossing changes needed to deform a diagram of $K$ into that of $K^{\prime}$, where the minimum is taken over all diagrams of $K$ from which one can obtain a diagram of $K^{\prime}$.

Then $d_{G}$ defines a metric on the space of the equivalence classes of knots or links. If $O$ is a trivial knot or link, then $d_{G}(K, O)$ is the unknotting or unlinking number of $K$, denoted by $u(K)$ (see [15]).

In this paper we determine the pairs of two-bridge knots or links with Gordian distance one. This result can be thought of as a generalization of those of KanenobuMurakami [7] and Kohn [8].

After having done this work, the author heard that J. Berge and I. DazeyD. W. Sumners had independently obtained a result similar to the main theorem, respectively (see [4]).

Throughout this paper we say that $K$ and $K^{\prime}$ are equivalent, denoted by $K=K^{\prime}$, if and only if there exists an orientation preserving homeomorphism of $S^{3}$ which maps $K$ to $K^{\prime}$.

\section{MAIN THEOREM}

Let $S(p, q)$ be the two-bridge knot or link whose two-fold branched cover is the lens space $L(p, q)$, where $p$ and $q$ are relatively prime. When $p$ is even, $S(p, q)$ is a two-component link, for $p$ odd, $S(p, q)$ is a knot.

$S(p, q)$ and $S\left(p^{\prime}, q^{\prime}\right)$ are equivalent if and only if $p=p^{\prime}$ and (I) $q \equiv q^{\prime}(\bmod p)$ or (II) $q q^{\prime} \equiv 1(\bmod p)[2$, Theorem $12.6(\mathrm{~b})]$.

Our main theorem is then the following.

Theorem 1. Let $S(p, q)$ and $S(r, s)$ be two-bridge knots or links. Then the following conditions are equivalent:

(i) $d_{G}(S(p, q), S(r, s))=1$.

Received by the editors April 8, 1996 and, in revised form, October 22, 1996.

1991 Mathematics Subject Classification. Primary 57M25.

(C)1998 American Mathematical Society 
(ii) There exist pairs of relatively prime integers $(m, n)$ and $(a, b)$ such that $n \neq$ $0, r m+a n \neq 0, r b-s a=1$ and $S(p, q)$ is equivalent to

$$
S\left(2 a n^{2}+r(2 m n \pm 1), 2 b n^{2}+s(2 m n \pm 1)\right) .
$$

(iii) There exist rational numbers $r_{1}$ and $r_{2}$ such that

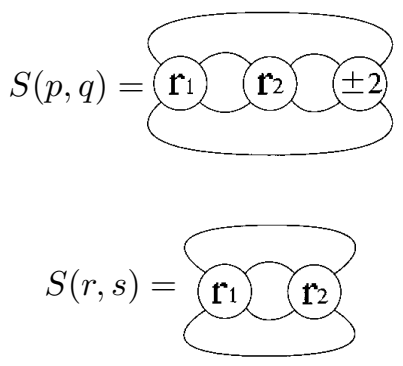

where see $[2$, Chapter 12]).

Remark 2. (i) $S(1,0)$ is a trivial knot. So the condition (ii) of Theorem 1 says that $d_{G}(S(p, q), S(1,0))=1$ if and only if

$$
\begin{aligned}
S(p, q) & =S\left(2 a n^{2}+2 m n \pm 1,2 n^{2}\right) \\
& =S\left(2 m^{\prime} n \pm 1,2 n^{2}\right)
\end{aligned}
$$

where $m^{\prime}=m+a n$. Therefore Theorem 1 is a generalization of KanenobuMurakami's theorem [7].

(ii) $S(0,1)$ is a trivial link. So the condition (ii) of Theorem 1 also says that $d_{G}(S(p, q), S(0,1))=1$ if and only if

$$
\begin{aligned}
S(p, q) & =S\left(-2 n^{2}, 2 b n^{2}+2 m n \pm 1\right) \\
& =S\left(-2 n^{2}, 2 m^{\prime} n \pm 1\right)
\end{aligned}
$$

where $m^{\prime}=m+b n$. Therefore Theorem 1 is also a generalization of Kohn's theorem $[8]$.

\section{Preliminaries}

Let $N(k)$ be a regular neighborhood of a knot $k$ in a closed orientable 3-manifold $M$, with $\mu$ a meridian of $N(k)$. Let $E(k)$ be the exterior of $k$ in $M$, that is, $E(k)=M-\operatorname{int} N(k)$. Now, let $k(\gamma)$ denote the closed manifold obtained by attaching a solid torus $V$ to $E(k)$ so that a curve of slope $\gamma$ on $\partial E(k)$ bounds a disk in $V$. Here the slope indicates the isotopy class of a nontrivial simple closed curve in $\partial E(k)$. We shall say that $k(\gamma)$ is the result of $\gamma$-surgery on $k$ in $M$. For two slopes $\gamma$ and $\delta$ in $\partial E(k)$, let $\Delta(\gamma, \delta)$ be their minimal geometric intersection number.

For oriented manifolds $M$ and $N, M \cong N$ means $M$ and $N$ are homeomorphic by an orientation preserving homeomorphism.

Lemma 3. If $d_{G}(S(p, q), S(r, s))=1$, then $L(p, q)$ is obtained by $\gamma$-surgery on some knot in $L(r, s)$, where $\Delta(\gamma, \mu)=2$. 
Proof. This is obtained by an argument similar to that of the proof of [9, Lemma 1] (cf. [7], [8]). In fact this follows from Montesinos' technique [11] and the fact that the double branched covering of $S^{3}$ along $S(r, s)$ is $L(r, s)$.

Let $m_{g}^{c}\left(\left(\alpha_{1}, \beta_{1}\right), \cdots,\left(\alpha_{t}, \beta_{t}\right)\right)$ be a Seifert fibred space, where $g$ is the genus of the orbit surface $F, c$ is the number of boundary components, and $t$ is the number of surgery instructions used to obtain the Seifert fibred space from the genuine (orientable) $S^{1}$-bundle over $F$. Each pair $\left(\alpha_{i}, \beta_{i}\right)$ specifies a particular surgery (for example, see [10, Chapter Four]). Our convention shall be that when $g$ is nonnegative, $F$ is orientable, while $g$ negative implies that $F$ is nonorientable $\left(F=\sharp_{i=1}^{|g|} R P^{2}\right)$. For later use, we note that $m_{g}^{c}\left(\left(\alpha_{1}, \beta_{1}\right), \cdots,\left(\alpha_{t}, \beta_{t}\right),(1, e)\right) \cong$ $m_{g}^{c}\left(\left(\alpha_{1}, \beta_{1}\right), \cdots,\left(\alpha_{i}, \beta_{i}+e \alpha_{i}\right), \cdots,\left(\alpha_{t}, \beta_{t}\right)\right)$ for any integer $e$ and any number $i$ $(1 \leq i \leq t)$.

Lemma 4. Let $k$ be a knot in $L(r, s)$. If $E(k)$ is a Seifert fibred space, then $k$ is a (possibly singular) fiber in some Seifert fibration of $L(r, s)$.

Proof. Let $V$ be a solid torus with meridian $\mu$. By hypothesis $E(k)$ is Seifert fibred and $L(r, s)$ is the union of $E(k)$ and $V$ along the boundary. The core of $V$ is isotopic to $k$ in $L(r, s)$. If $E(k)=m_{g}^{1}\left(\left(\alpha_{1}, \beta_{1}\right), \cdots,\left(\alpha_{t}, \beta_{t}\right)\right)$, then by using the fact that $H_{1}(k(\mu))$ is a cyclic group (cf. [8]), we see $g=0$ or -1 . There are also two cases to consider: $\mu$ is either identified with a fiber of $E(k)$ or not. If $\mu$ is not a fiber, then the Seifert fibration extends on the resulting manifold and $k$ is a fiber. Therefore hereafter we assume $\mu$ is a fiber. First we assume $g=0$, then $t \leq 1$ because if otherwise $L(r, s)$ has a separating essential 2 -sphere by a standard argument (see [6]), a contradiction. Therefore in this case $E(k)$ is a solid torus. Hence the statement follows immediately by re-fibering $E(k)$. Second we assume $g=-1$. Then $t \leq 0$ by a standard argument as above. Then $E(k)$ is a twisted $S^{1}$-bundle over a Möbius band and it is homeomorphic to $S^{2} \times S^{1}$ minus a regular neighborhood of a "(2,1)-torus knot" (see [8, Lemma 4]), and this is homeomorphic to a twisted annulus bundle over a circle. And the fibers are parallel circles on the annuli. Therefore as in [8, Lemma 4], the statement of Lemma 4 follows by re-fibering $E(k)$.

Lemma 5 (The classification of the Seifert fibration for a lens space). Suppose $m_{g}^{0}\left(\left(\alpha_{1}, \beta_{1}\right), \cdots,\left(\alpha_{t}, \beta_{t}\right)\right)\left(\alpha_{i} \geq 2\right)$ is a lens space. Then one of the following conditions holds.

(1) $g=0$ and $t \leq 2$,

(2) $g=-1$ and $t \leq 1$.

Proof. This can be proved by a standard argument as in the proof of Lemma 4 .

Lemma 6. If $d_{G}(S(p, q), S(r, s))=1$, then for some Seifert fibering of $L(r, s)$, $L(p, q)$ is obtained by $\gamma$-surgery along a fiber, where $\Delta(\gamma, \mu)=2$.

Proof. From Lemma 3 we know that $L(p, q)$ is obtained by $\gamma$-surgery on some knot $k$ in $L(r, s)$, where $\Delta(\gamma, \mu)=2$. So both $\gamma$ and $\mu$ are cyclic slopes; that is, $\gamma$ and $\mu$ surgery yield the manifold with cyclic fundamental groups. By the Cyclic Surgery Theorem [3], $E(k)$ is reducible or Seifert fibred. If $E(k)$ is reducible, then $k(\gamma)$ has $L(r, s)$ as a connected summand. Hence $L(p, q)=k(\gamma)=L(r, s)$. This contradicts our assumption. Therefore $E(k)$ is a Seifert fibred space. Lemma 4 implies that $k$ is a fiber in some Seifert fibration of $L(r, s)$. 
Now we describe surgeries on fibers of Seifert fibrations of lens spaces. For $i=1,2$, let $V_{i}$ be a solid torus standardly embedded in $S^{3}$ and let $\mu_{i}$ and $\lambda_{i}$ be a meridian and a longitude of $V_{i}$ respectively. Let $h$ be an orientation-reversing homeomorphism from $\partial V_{1}$ to $\partial V_{2}$ such that $h\left(\mu_{1}\right)=s \mu_{2}+r \lambda_{2}$. Then the space $V_{1} \cup_{h} V_{2}$ obtained from $V_{1}$ and $V_{2}$ by identifying their boundaries by $h$ is the lens space $L(r, s)$.

Let $C_{m, n}$ be a $(m, n)$-curve on $\partial V_{1}$, that is, a simple loop on $\partial V_{1}$ which is isotopic to $m \mu_{1}+n \lambda_{1}$. Let $a$ and $b$ be integers such that $r b-s a=1$. Then we may assume $h\left(\lambda_{1}\right)=b \mu_{2}+a \lambda_{2}$ and $C_{m, n}$ is isotopic to $(s m+b n) \mu_{2}+(r m+a n) \lambda_{2}$ on $\partial V_{2}=\partial V_{1}$. $L(r, s)$ has a Seifert fibration in which $C_{m, n}$ is a fiber if and only if $n \neq 0$ and $r m+a n \neq 0$. In fact, such a Seifert fibration is given by $m_{0}^{0}((n, x),(r m+a n, y))$, where $x$ and $y$ are some integers (cf. [8, p.1137]). We may push $C_{m, n}$ into int $V_{1}$. Then, for a slope $\gamma$ on $\partial N\left(C_{m, n}\right)$, using the usual meridian-longitude coordinates of $\partial N\left(C_{m, n}\right)$ in $V_{1} \subset S^{3}$, we identify $\gamma$ with $c / d \in \mathbb{Q} \cup\{\infty\}$, with $c$ and $d$ relatively prime. Now we perform $c / d$-surgery on $C_{m, n}$ in $V_{1} \subset L(r, s)$.

Lemma 7. Let $L(r, s) \supset C_{m, n}$ be as above. Then

(i) $C_{m, n}(c / d) \cong m_{0}^{0}((n, x),(r m+a n, y),(c-d m n, d))$, where $L(r, s) \cong$ $m_{0}^{0}((n, x),(r m+a n, y))$ and $a, x, y$ are as above.

(ii) If $c=d m n \pm 1$, then $C_{m, n}(c / d) \cong L\left(d a n^{2}+r(d m n \pm 1), d b n^{2}+s(d m n \pm 1)\right)$, where $a$ and $b$ are as above.

Proof. (i) This is obtained by using the fact that an ordinary fiber on $\partial N\left(C_{m, n}\right) \subset$ $m_{0}^{0}((n, x),(r m+a n, y))$ has slope $m n$ (cf. [12]).

(ii) By [5, Lemma 7.2 and its proof], if $c=d m n \pm 1$, then after surgery $V_{1}$ changes into another solid torus $V_{1}^{\prime}$ with meridian $(d m n \pm 1) \mu_{1}+d n^{2} \lambda_{1}$. Since the image of the meridian of $V_{1}^{\prime}$ by $h$ is

$$
\begin{aligned}
\left(h\left(\mu_{1}\right) \quad h\left(\lambda_{1}\right)\right)\left(\begin{array}{c}
d m n \pm 1 \\
d n^{2}
\end{array}\right) & =\left(\begin{array}{ll}
\mu_{2} & \lambda_{2}
\end{array}\right)\left(\begin{array}{ll}
s & b \\
r & a
\end{array}\right)\left(\begin{array}{c}
d m n \pm 1 \\
d n^{2}
\end{array}\right) \\
& =\left(\begin{array}{ll}
\mu_{2} & \lambda_{2}
\end{array}\right)\left(\begin{array}{c}
d b n^{2}+s(d m n \pm 1 \\
d a n^{2}+r(d m n \pm 1
\end{array}\right)
\end{aligned}
$$

we obtain the desired result.

\section{Proof of Theorem 1}

(i) $\Rightarrow$ (ii). By Lemma 6 , there is a fiber $k$ of $L(r, s)$ such that $\gamma$-surgery along $k$ results in $L(p, q)$, where $\Delta(\gamma, \mu)=2$. By Lemma 5 , the Seifert fibration is over $S^{2}$ or $R P^{2}$. First we consider the $S^{2}$ case. Then $k$ is isotopic to some $C_{m, n} \subset$ $\partial V_{1}=\partial V_{2} \subset V_{1} \cup_{h} V_{2}=L(r, s)$, where $V_{1} \cup_{h} V_{2}$ is as in Section 3. Since $k=C_{m, n}$ is a fiber, $n$ and $r m+a n$ are nonzero. Suppose $|n|$ and $|r m+a n| \geq 2$. Then $\gamma=c / 2$ for some odd integer $c$, because $\Delta(\gamma, \mu)=2$. By Lemma 7 (i), $L(p, q) \cong$ $m_{0}^{0}((n, x),(r m+a n, y),(c-2 m n, 2))$, where $L(r, s) \cong m_{0}^{0}((n, x),(r m+a n, y))$. Then, since $|n|,|r m+a n| \geq 2$, we have $c-2 m n=\epsilon$ where $\epsilon= \pm 1$ by Lemma 5 . So by Lemma 7 (ii), $L(p, q) \cong L\left(2 a n^{2}+r(2 m n \pm 1), 2 b n^{2}+s(2 m n \pm 1)\right)$. Therefore $S(p, q)$ is equivalent to $S\left(2 a n^{2}+r(2 m n \pm 1), 2 b n^{2}+s(2 m n \pm 1)\right)$, where $(m, n)$ and $(a, b)$ satisfy the condition of the statement (ii). Secondly we consider the case where $|n|=1$ or $|r m+a n|=1$. Here, without loss of generality, we can assume $|n|=1$. Then if $c=2 m^{\prime}+1$ for some integer $m^{\prime}$, we can put $k=C_{m^{\prime}, 1}$. Therefore as in the case $n \geq 2$, by Lemma 7 (ii), the statement follows. 
Next we consider the $R P^{2}$ case. Then $L(r, s)=E(k) \cup_{i d} N(k)$ and $L(p, q)=$ $E(k) \cup_{h} N(k)$, where $\Delta(h(\mu), \mu)=2$. If $k$ is an ordinary fiber, then the core of $N(k) \subset E(k) \cup_{h} N(k)$ is a singular fiber, since $\Delta(h(\mu), \mu)=2$. Therefore by Lemma $5, E(k)$ is a twisted $S^{1}$-bundle over a Möbius band whether $k$ is a singular fiber or not. As in the proof of Lemma $4, L(r, s)$ and $L(p, q)$ are obtained by surgery along a "(2,1)-torus knot" in $S^{2} \times S^{1}$. Therefore, by re-fibering $E(k)$, we see $L(r, s) \cong$ $m_{0}^{0}((2,1),(2,-1),(1, d))$ and $L(p, q) \cong m_{0}^{0}((2,1),(2,-1),(1, d \pm 2))$ for some $d$ (cf. [8, p.1139]). Hence, by changing invariants $L(r, s) \cong m_{0}^{0}((2,1),(2,2 d-1))$ and $L(p, q) \cong m_{0}^{0}((2,1),(2,2 d-1),(1, \pm 2))$. Then $L(p, q)$ is obtained by $c / 2$-surgery along $C_{2, z} \subset V_{1} \cup V_{2} \subset L(r, s)$ for some $z$, where $h\left(\mu_{1}\right)=r \lambda_{2}+s \mu_{2}, h\left(\lambda_{1}\right)=$ $a \lambda_{2}+b \mu_{2}, r b-s a=1$ and $c=4 z \pm 1$. Therefore as in the $S^{2}$ case, $(p, q)$ has the desired description.

(ii) $\Rightarrow$ (iii). As above $L(p, q)$ can be obtained by $c / 2$-surgerying along $C_{m, n} \subset$ $V_{1} \subset V_{1} \cup_{h} V_{2}=L(r, s)$, where $V_{1} \cup_{h} V_{2}$ is as in Section 3. Then $L(p, q) \cong$ $m_{0}^{0}((n, x),(r m+a n, y),( \pm 1,2))$, where $L(r, s) \cong m_{0}^{0}((n, x),(r m+a n, y))$ similarly. Hence $L(p, q)$ and $L(r, s)$ are the double covers of the Montesinos knots or links (see [2, Chapter 12]) as in the statement (iii) of Theorem 1 , where $r_{1}=x / n$ and $r_{2}=y /(r m+a n)$. Therefore $S(p, q)$ and $S(r, s)$ have the desired description.

(iii) $\Rightarrow$ (i).

Since $\underset{+2}{+}=\mathcal{C}$ or $\gamma^{\prime}$, we can easily see $d_{G}(S(p, q), S(r, s)) \leq 1$.

It remains to prove $d_{G}(S(p, q), S(r, s)) \neq 0$. Suppose $S(p, q)$ is not equivalent to $S(r, s)$. Then applying the above arguments to a crossing change in +2 by (ii), there exist pairs of relatively prime integers $(m, n)$ and $(a, b)$ such that $n \neq 0, r m+a n \neq 0, r b-s a=1 S\left(2 a n^{2}+r(2 m n \pm 1), 2 b n^{2}+s(2 m n \pm 1)\right)$ is equivalent to $S(r, s)$. Therefore it follows that $r=2 a n^{2}+r(2 m n \pm 1)$ and (I) $s \equiv 2 b n^{2}+s(2 m n \pm 1)(\bmod r)$ or (II) $s\left(2 b n^{2}+s(2 m n \pm 1)\right) \equiv 1(\bmod r)$. But under the above conditions, elementary number theory for the congruences easily proves these cases never occur. This makes a contradiction.

This completes the proof of Theorem 1.

\section{Addendum}

In this section we measure the Gordian distance between a Montesinos knot (or link) and a two-bridge knot (or link). Though the proof of Theorem 1 depends on the Cyclic Surgery Theorem, that of the following theorem will depend on the recent Boyer-Zhang's theorem [1].

Theorem 8. Let $K=M\left(\left(\alpha_{1}, \beta_{1}\right), \cdots,\left(\alpha_{t}, \beta_{t}\right)\right)$ be a Montesinos knot or link with $\alpha_{i} \geq 2, t \geq 4$. Then $d_{G}(K, S(p, q)) \geq 2$, for any two-bridge knot or link $S(p, q)$.

Remark 9. In [14] Motegi independently proved Theorem 8 for the knot case.

Proof of Theorem 8 (cf. [13], [17]). We assume that $d_{G}(K, S(p, q))=1$. Then by analogy of Lemma 3, the double cover of $M_{K}$ of $K$ is obtained by $\gamma$-surgery on some knot $k$ in $L(p, q)$, where $\Delta(\gamma, \mu)=2$. Here $M_{K}=m_{0}^{0}\left(\left(\alpha_{1}, \beta_{1}\right), \cdots,\left(\alpha_{t}, \beta_{t}\right)\right)$. We remark that $E(k)$ is irreducible. Because if otherwise, then $k(\gamma)$ must be a reducible Seifert fibred space. This contradicts our assumption. Hence by BoyerZhang's result [1, Theorem I] $E(k)$ is a Seifert fibred space or a cable on a Seifert fibred space. 
If $E(k)$ is the former, then, by Lemma $4, k$ is a fiber for some Seifert fibration of $L(p, q)$. Then, by an argument as in Section 3, we can prove $k(\gamma)$ can never be such a Seifert fibred space as the number of singular fibers $\geq 4$. If $E(k)$ is the latter, then there exists a cable space $C$ such that $\partial C=T_{1} \amalg T_{2}$, where $\partial E(k)=T_{1}$ and

$$
E(k)=m_{g}^{1}\left(\left(\alpha_{1}, \beta_{1}\right), \cdots,\left(\alpha_{u}, \beta_{u}\right)\right) \cup_{T_{2}} C
$$

for some integers $g$ and $u$. For any slope $\delta$ on $T_{1}=\partial E(k)$, let $C(\delta)$ denote the manifold obtained by $\gamma$-surgery on $k$ in $C$. Since $k(\mu)=L(p, q), C(\mu)$ must be a solid torus. Let $k^{\prime}$ be a core of $C(\mu)$. We can regard $k^{\prime}$ as a knot in $L(p, q)$. Then

$$
E\left(k^{\prime}\right)=m_{g}^{1}\left(\left(\alpha_{1}, \beta_{1}\right), \cdots,\left(\alpha_{u}, \beta_{u}\right)\right) .
$$

Again by Lemma $4, k^{\prime}$ is a fiber for some Seifert fibration of $L(p, q)$. Hence we can also prove that $k(\gamma)=E\left(k^{\prime}\right) \cup C(\mu)$ cannot be such a Seifert fibred space.

This makes a contradiction.

\section{ACKNOWLEDGMENTS}

The author would like to thank K. Kawakubo for his kind encouragement. He is also grateful for several valuable conversations concerning the material of this paper offered by M. Sakuma.

\section{REFERENCES}

1. Boyer, S. and Zhang, X., Exceptional surgery on knots, Bull. AMS (2) 31 (1994), 197-203. MR 95f: 57035

2. Burde, G. and Zieschang, H., Knots, de Gruyter Studies in Mathematics, no. 5, Walter de Gruyter, Berlin, 1985. MR 87b:57004

3. Culler, M., Gordon, C.McA., Luecke, J. and Shalen, P., Dehn surgery on knots, Ann. of Math. (2) $\mathbf{1 2 5}$ (1987), 237-300. MR 88h:57026

4. I. Dazey and D. W. Sumners, A strand passage metric for topoisomerase action, Proceedings of Knots 96 (ed. by S. Suzuki), World Scientific Publishing Co. (1997), 267-278.

5. Gordon, C.McA., Dehn surgery and satellite knots, Trans. Amer. Math. Soc. 275 (1983), 687-708. MR 84d:57003

6. Heil, W., 3-manifolds that are sums of solid tori and Seifert fiber spaces, Proc. Amer. Math. Soc. 37 (1973), 609-614. MR 50:8526

7. Kanenobu, T. and Murakami, H., Two-bridge knots with unknotting number one, Proc. Amer. Math. Soc. 98 (1986), 499-502. MR 87i:57005

8. Kohn, P., Two-bridge links with unlinking number one, Proc. Amer. Math. Soc. 113 (1991), 1135-1147. MR 92c:57008

9. Lickorish, W.B.R., The unknotting number of a classical knot, Contemp. Math., vol. 44, Amer. Math. Soc., Providence, RI, 1985, pp. 117-121. MR 87a:57012

10. Montesinos, J.M., Classical Tessellations and Three-Manifolds, Springer-Verlag, 1987. MR 89d:57016

11. Montesinos, J.M, Surgery on links and double branched coverings of $S^{3}$, Ann. of Math. Studies 84 (1975), 227-259. MR 52:1699

12. Moser, L., Elementary surgery along a torus knot, Pacific J. Math. 38 (1971), 737-745. MR 52: 4287

13. Motegi, K., A note on unlinking numbers of Montesinos links, Rev. Mat. Complut. Madrid 9 (1996), 151-164. CMP 97:02

14. Motegi, K., Bridge numbers of twisted Montesinos knots, Proceedings of The Institute of Natural Sciences, Nihon University No. 31 (1996).

15. Murakami, H., Some Metrics on Classical Knots, Math. Ann. 270 (1985), 35-45. MR 86g:57007

16. Rolfsen, D., Knots and links, Mathematics Lecture Series, no.7, Publish or Perish, Berkeley, CA, 1976. MR 58:24236 
17. Torisu, I., A note on Montesinos links with unlinking number one (conjectures and partial solutions), Kobe J. of Math. 13 (1996), 167-175.

18. I. Torisu, On nugatory crossings for knots, to appear in Topology and its Appl.

Department of Mathematics, Osaka University, Toyonaka, Osaka, 560, Japan

E-mail address: torisu@math.sci.osaka-u.ac.jp 